\title{
26. Bemerkungen von Gen. N. S. Chruschtschow vom 21. Mai 1958 zum Entwurf einer Rede ${ }^{468}$
}

- Ich würde es so sagen, alle Länder des aggressiven NATO-Blocks haben jetzt zu berücksichtigen, dass die Entwicklung der Wissenschaft und die Entwicklung der Technik jetzt gleiche Bedingungen für alle industrialisierten und hoch entwickelten Länder bietet, die vollkommensten Vernichtungsinstrumente zu schaffen. Wenn daher die USA ihre Stützpunkte näher an unsere Territorien heranrücken, sollte jedem kultivierten Menschen mit Kenntnissen über die Entwicklung von Wissenschaft und Technik klar sein, dass die Sowjetunion und die anderen Länder des Warschauer Zusammenschlusses die gleichen Mittel haben können oder schon haben. Deswegen sollte man jetzt nicht von der „Position der Stärke" ausgehen, und dass die eine Seite die anderen einschüchtert. Dies führt nur zum Wettrüsten, und jedes Wettrüsten endet in einem Vernichtungskrieg. Davon, dass die Länder des Warschauer Pakts moderne Mittel besitzen, zu denen jedes Industrieland mit hoher Kultur, Wissenschaft und Technik Zugang hat, legt der Abschuss unserer Erdsatelliten ${ }^{469}$ und unserer ballistischen Raketen Zeugnis ab. Deswegen sollte jeder vernünftige Mensch nüchtern an die Lage herangehen und sie richtig einschätzen und seinerseits alles dafür tun, um die Atmosphäre nicht anzuheizen, keine Umstände des Wettrüstens und der Annäherung an den Krieg zu schaffen, sondern alles daran setzen, um den Kalten Krieg $\mathrm{zu}$ liquidieren und wirklich $\mathrm{zu}$ gutnachbarlichen Beziehungen zwischen allen Ländern überzugehen.

- Es hat gewisse Erklärungen gegeben mit Gutachten amerikanischer Ärzte zur psychischen Verfassung. 470

- Diese Vorschläge machen lediglich klar, dass die Vereinigten Staaten von Amerika ihre Politik „aus einer Position der Stärke heraus“ fortsetzen, und das können sie nur den Staaten vorschlagen, die auf diesem Standpunkt stehen, doch sie täuschen sich. Gegen jede Kraft wirkt eine Gegenkraft, und deswegen kann kein Staat, zumal wenn er über die Mittel zur Verteidigung seines Territoriums ver-

468 Die Worte: „zum Entwurf einer Rede“ wurden handschriftlich hinzugefügt. Bei dem Text, auf den sich die nachstehenden Bemerkungen beziehen, handelt es sich vermutlich um einen Entwurf, den man Chruščëv als Grundlage für eine weitere Stellungnahme auf der Beratung der kommunistischen und Arbeiterparteien der sozialistischen Länder in Moskau vom 20. bis 23. Mai 1958 vorgelegt hatte. Ein Anstrich am Anfang eines Absatzes in den nachstehenden Bemerkungen zeigt an, dass sich Chruščëv zu einem weiteren Teil des - der Nachwelt in den Akten nicht überlieferten - Entwurfs äußerte.

469 Am 4. Oktober 1957 hatte die UdSSR den ersten künstlichen Erdsatelliten gestartet, dem inzwischen noch zwei gefolgt waren.

4702000 amerikanische Wissenschaftlern am 3. Juni 1957 in einem - von Linus Pauling initiierten Appell den sofortigen Abschluss eines Abkommens zur Beendigung der Kernwaffenversuche wegen der durch diese Tests hervorgerufenen gesundheitlichen Schäden gefordert hatten. Das hatte in der Öffentlichkeit eine Diskussion ausgelöst, in deren Verlauf Genetiker am 4. Juni 1957 vor einem Kongresskomitee aussagten, es gebe nichts, was man als ungefährliche Bestrahlungsdosis ansehen könnte. Es sei daher zu erwarten, dass die jetzt der Radioaktivität ausgesetzten Menschen etwa 2 Mrd. Kinder zur Welt bringen würden, von denen möglicherweise 80000 erhebliche genetisch bedingte geistige und physische Defekte hätten. Vielleicht hatten auch amerikanische Ärzte im Blick auf die seit 1957 in jedem Jahr stattfindende internationale Pugwash-Konferenz ein derartiges Gutachten verfasst. 
fügt, die einseitig vorteilhaften Vorschläge akzeptieren, die von den Vereinigten Staaten von Amerika unterbreitet werden.

- Bei der Vorlage ihrer Vorschläge sichern sie [die USA] nicht einmal zu, die Flüge völlig zu beenden, sondern sie nur zu verringern. ${ }^{471}$

- Hier ist zu sagen, dass die Vereinigten Staaten von Amerika mit ihrer Politik des Aufbaus von Militärstützpunkten auf fremdem Territorium schon viel früher begonnen haben, bevor überhaupt Raketen, zumal interkontinentale Raketen, geschaffen worden waren.

- „In die Enge treiben“, das ist etwas ungenau. Sie haben nicht nur in die Enge treiben wollen, sondern sie möchten die von den Völkern in den sozialistischen Ländern errichtete sozialistische Ordnung liquidieren. Jetzt aber reden sie davon, dass sich dies ihr Bestreben nicht auf die Sowjetunion beziehe, sondern es beziehe sich auf die, wie sie sie nennen, osteuropäischen Länder. Doch das ist schon nicht mehr das Ergebnis eines gesunden Urteils, eines richtigen Verständnisses der Lage, sondern sie wollen diese Aufgabe in Etappen lösen und berücksichtigen dabei, dass sie nicht über die Mittel verfügen, um die grundlegenden Aufgaben zu lösen, die sie sich stellen. Doch sie finanzieren die Kräfte, die sowohl gegen die Sowjetunion als auch gegen alle sozialistischen Länder agieren.

- Sollten wir denn das sagen? Das wärmt die Sache [bloß] auf. Sie hinken hinterher, hinken ernstlich hinterher - darum geht es nicht. Das versteht sich von selbst. Wir sollten jetzt unsere Überlegenheit in der Raketentechnik nicht hervorkehren. Da sie hinterherhinken, werden sie auf Sympathie für [den Wunsch] stoßen, dass man uns einholen müsse. ${ }^{472}$ Schließlich haben viele Angst vorm Kommunismus. Daher sollten wir nicht auf dieser Grundlage einen Wettstreit in Gang setzen.

- Und besonders lässt sich das sehen nach dem Abschuss der drei sowjetischen Satelliten in den kosmischen Raum, die den erreichten Entwicklungsstand der Industrialisierung der Sowjetunion und der Entwicklung von Wissenschaft und Technik demonstriert haben, dass sie auf dem höchsten Stand sind, der, was wir nicht in Abrede stellen, ebenso sowohl von den Vereinigten Staaten von Amerika als auch von anderen industriell hoch entwickelten Ländern erreicht werden kann.

- Doch einseitige Maßnahmen lassen sich verständlicherweise nicht unendlich fortsetzen. Das geht nicht. Was bringt es uns? Das heißt, wir beginnen mit Aufrüstung und Krieg. Welchen Weg zurück gibt es denn? Dass wir auch weiter beharrlich diese Politik fortsetzen werden, um Klärung herbeizuführen, und mit jedem Tag werden mehr und mehr die Völker aller Länder die friedliebende sow-

471 Gemeint sind die amerikanischen Aufklärungsflüge über der UdSSR, die seit langem erfolgten, ohne dass deren Luftverteidigung es verhindern konnte. Nach amtlicher Auffassung in Washington dienten die Flüge vor allem der Feststellung der sowjetischen Raketen, und waren deswegen auch im Kontext der angestrebten Abrüstung beider Seiten notwendig, wie Präsident Eisenhower zur Begründung seines Vorschlags der „offenen Himmel“ (Open Skies) am 21. Juli 1956 auf der Genfer Gipfelkonferenz ausgeführt hatte. Intern hieß es, man müsse den Nachteil, den man als Demokratie gegenüber einer Diktatur beim Einsatz von Spionen habe, durch den Einsatz technischer Aufklärungsmittel kompensieren.

472 Die USA hatten mit breiter Unterstützung der Öffentlichkeit Anstrengungen zur Überwindung des Rückstands eingeleitet. 
jetische Politik begreifen und sie richtig bewerten. Und deswegen werden wir mit unserer beharrlichen Arbeit erreichen, dass die Völker der Länder, deren Regierungen eine Politik „aus einer Position der Stärke heraus“ und des Wettrüstens betreiben, ihre Regierungen dazu zwingen werden, sich auf diesen Standpunkt [der UdSSR] zu stellen.

Hier würde ich meinen, ist es vielleicht angebracht, folgenden Gedanken zu äußern: Jetzt läuft die Taktik der Vereinigten Staaten von Amerika und der Länder, die sie unterstützen, darauf hinaus, in Erklärungen und in Gesprächen durch eingehende Vorbereitung der Gipfelkonferenz und durch [das Verlangen nach] Gewährleistung positiver Beschlüsse seitens dieser Konferenz die Aufmerksamkeit einzuschläfern, die Beratungen darüber in die Länge zu ziehen, um die öffentliche Meinung bei ihrer Forderung nach einer möglichst raschen Einberufung der Konferenz zu bremsen und diese zuletzt für immer zu begraben. ${ }^{473}$ Und das Gerede, wesentlich sei der Gedanke einer sorgfältigen Vorbereitung [der Konferenz mit der UdSSR], das heißt die Durchführung einer Politik „aus der Position der Stärke heraus“, um die Sowjetunion und die Länder des Warschauer Zusammenschlusses zu nötigen, die Forderungen der USA ohne Widerrede zu akzeptieren, wie sie selbst offen sagen. Wer kann denn wirklich im Ernst meinen, dass sich aufgrund einer sorgfältigen Vorbereitung dieser Gipfelkonferenz Fragen regeln lassen ${ }^{474}$ wie die Einmischung in die [Angelegenheiten der] osteuropäischen Länder mit dem Ziel der Veränderung des Regimes, das jetzt sozialistisch errichtet worden ist, oder aber die Vereinigung Deutschlands unter Absehung vom Bestehen zweier souveräner deutscher Staaten, ohne sie und hinter ihrem Rücken im Interesse nur eines deutschen Staates - Deutschlands 475 - natürlich sind das keine seriösen Gespräche. Und wenn es darum geht, die Zeit dadurch hinauszuzögern, dass man sich auf eine sorgfältige Vorbereitung beruft, dann wird es offensichtlich kein Ende bei der Vorbereitungszeit geben, weil die Sowjetunion so einer Regelung unter keinen Umständen zustimmen kann und nicht darauf eingehen wird. Doch die Sowjetunion hat konkrete Vorschläge vorgelegt, und diese Vorschläge wurden vom Leben selbst formuliert. Deswegen kann man hier beliebige Fragen auswählen und regeln und damit den Anfang zur Errichtung des Gebäudes machen, über das man gesprochen hat.

${ }^{473}$ Am 8. Januar 1958 hatte die sowjetische Regierung den Mitgliedstaaten der UNO, der Bundesrepublik Deutschland und der Schweiz in einem veröffentlichten Memorandum die baldige Einberufung einer Konferenz der Regierungschefs aller interessierten Mächte vorgeschlagen, auf der ein Moratorium der Kernwaffenversuche für die Dauer von ein bis zwei Jahren, ein allgemeines Verbot der Nuklearwaffen, die Schaffung einer atomwaffenfreien Zone in Mitteleuropa, der Abschluss eines Nichtangriffspakts zwischen den Staaten der NATO und des Warschauer Pakts, eine Reduzierung bzw. der Abzug der auf dem Gebiet Deutschlands und anderer europäischer Staaten stationierten Truppen, ein Abkommen zur Verhinderung von Überraschungsangriffen, einer Erweiterung der internationalen Handelsbeziehungen, eine Unterbindung der Kriegspropaganda und die Verringerung der Spannungen im Nahen Osten erörtert werden sollten. Diese Themen wurden zum Gegenstand einer breiten Propagandakampagne, welche die Abhaltung der Gipfelkonferenz forderte. Während die westlichen Staaten eine skeptische Haltung einnahmen und allenfalls zu einer Vier-Mächte-Konferenz bereit waren, bemühte sich die UdSSR vor allem bei den Ländern der Dritten Welt um Unterstützung ihres Vorschlags.

${ }_{474} \mathrm{Im}$ - augenscheinlich sehr schnell notierten - russischen Text fehlt die hier eingefügte grammatikalische Satzaussage.

475 So im russischen Text, ohne dass es klar wird, was dieses Wort hier soll. 
- Daher stehen wir auf dem Standpunkt, dass bei einer Zusammenkunft davon auszugehen und die Regelung solcher Fragen vorzubereiten ist, die möglich sind und vielleicht geregelt werden können, und die Fragen lassen sich nur regeln, wenn die Länder mit verschiedenem Aufbau im Innern, das heißt die kapitalistischen und die sozialistischen Länder, real[istisch] an die Sache herangehen und das Bestehen zweier Weltsysteme - des Kapitalismus und des Sozialismus - akzeptieren, [wenn sie] die Anerkennung dieser Koexistenz und den Wunsch zum Ausdruck bringen, ${ }^{476}$ die friedliche Koexistenz zu gewährleisten und die Fragen zu regeln, die zur friedlichen Koexistenz beitragen. Deswegen haben wir gerade die Fragen ausgewählt, die nicht die Fundamente der kapitalistischen Länder berühren und auch nicht die Interessen der sozialistischen Länder berühren. Das ist der Dreh- und Angelpunkt. Wir schüchtern einander nicht ein, aber...

- Sollte man hier nicht unseren Gedanken zum Ausdruck bringen. Wir haben darauf seit langem verzichtet, doch wir haben ihn benutzt, als wir die Frage aufwarfen, die Produktion von Kernwaffen unter Kontrolle zu stellen. Das war der Fall beim Vorschlag der Amerikaner, die Entwicklung der Kernenergie gestatte es nunmehr, Energie für friedliche $Z$ wecke einzusetzen, und zugleich werden dabei nukleare Instrumente geschaffen, die sich für militärische Zwecke benutzen lassen. Mithin ist es bereits jetzt unmöglich, eine absolute Kontrolle zu erreichen, weil die Produktion der Kernenergie und ihre Verwendung für friedliche Zwecke zugleich die Produktion der gleichen Kernenergie für militärische Zwecke ist, und bereits die Herstellung militärischer Instrumente, das heißt die Konstruktion von Bomben für Flugzeuge und Raketen - das ist schon keine so schwierige Sache mehr unter dem Gesichtspunkt, dass irgendwer die Produktion dieser Kernwaffe geheim halten möchte, und das lässt sich heute kaum noch einer Kontrolle unterwerfen. Darum sind diese Umstände, die wir haben, real zu berücksichtigen. Deswegen besteht der einzige Ausweg in dieser Frage darin, ihren [der nuklearen Instrumente] Gebrauch moralisch zu verurteilen, und das wird sie davon abhalten, denn dafür gibt es Beispiele, und das umso mehr, als diese Instrumente in diesem wie jenem Staat zu finden sein werden. Und darum wird das ein Faktor der Zügelung hinsichtlich des Gebrauchs diese Instrumente sein, und am wichtigsten ist die moralische Verpflichtung, die es geben wird. Das ist der Beginn, danach aber, wenn die Beziehungen sich entwickeln und festigen [und] in freundschaftliche Beziehungen zwischen den Ländern übergehen, werden günstigere Bedingungen für eine breitere Kontrolle und für das Vertrauen zwischen den Ländern entstehen, und auf diese Weise lässt sich der Krieg als Mittel zur Regelung von Streitfragen ausschließen.

- Die Hauptsache, vielleicht sollte man das jetzt nicht sagen. Suchen Sie die Formulierung herauszunehmen.

- Was ich diktiert habe, passt hierher.

- Hier sollte irgendwo gesagt werden, dass diese Vorschläge sowohl den Interessen der Völker der sozialistischen Länder entsprechen als auch den Interessen 
der Völker der Länder entsprechen, die den aggressiven Blöcken des Westens der NATO, der SEATO, 477 dem Bagdad-Pakt ${ }^{478}$ u. a. - angehören.

- Hier im vorletzten Absatz dennoch [ausführen], dass wir keinen Angriff vorhaben, aber einen kühlen Kopf behalten und die Verantwortung für die Sicherheit unserer sozialistischen Länder angesichts einer ganzen Reihe von Schritten übernehmen müssen, die von unseren sozialistischen Ländern zur Beendigung des Kalten Kriegs, zur Reduzierung der Streitkräfte und zur Herstellung der Bedingungen für eine friedliche Koexistenz unternommen wurden. Sie haben bisher nicht nur keine Antwort von den zur NATO gehörenden Ländern erhalten, sondern es wird alles dafür getan, um sie zunichte zu machen und die Atmosphäre des Wettrüstens weiter anzuheizen. Daher können wir keine Situation zulassen, bei der wir die Wachsamkeit einbüßen und Bedingungen dafür schaffen würden, dass die Politik „aus einer Position der Stärke heraus“ die Möglichkeit erhielte, diese Stärke gegen unseren Staat zu richten. Deswegen sollten wir alles für die internationale Entspannung tun, doch wir müssen [auch] alle Bedingungen dafür schaffen, dass die Politiker, die sich auf die Politik „aus einer Position der Stärke heraus" und eine Politik am Rande des Krieges ${ }^{479}$ stützen, wissen, wenn sie diesen Krieg entfesseln, werden sie in diesem Krieg eine Niederlage erleiden. Das muss man sagen.

Die Anhänger der Politik „aus einer Position der Stärke heraus“ und der Verstärkung des Kalten Krieges und auf dieser Basis der vermehrten Produktion von Vernichtungsinstrumenten suchen der Arbeiterklasse und der Intelligenz unzweideutig den Gedanken aufzunötigen, dass so eine Politik in gewissem Umfang auch der Arbeiterklasse zugute komme, weil sie die Bedingungen schaffe für mehr Beschäftigung in der Industrie, in der Produktion. Und darum suchen sie sogar damit einzuschüchtern, dass im Falle der Beendigung des Kalten Krieges und einer Übereinkunft über friedliche Koexistenz die Notwendigkeit des Wettrüstens entfiele, es folglich zur Einschränkung der Produktion und zur Vergrößerung der Armee der Arbeitslosen, zum Sinken des Lebensstandards der Werktätigen käme.

Sind derartige Argumente richtig, welche die Gegner der Beendigung des Kalten Krieges und die Lakaien des Monopolkapitals in die Welt zu setzen versuchen? Nein, sie sind nicht richtig, weil es den fundamentalen Grundlagen des menschlichen Wesens widerspricht, weil es die Produktion der Vernichtungsinstrumente erhöht, es widerspricht allem Lebendigen, weil alles Lebendige um sein Weiterleben kämpft, hier jedoch schafft der Mensch selbst die Vernichtungsinstrumente. Zudem, wenn man es unter dem Blickwinkel dieser Argumente des ho-

477 Nach dem Sieg der vietnamesischen Kommunisten über die französische Kolonialmacht und der Gründung ihres Staates Nordvietnam auf der internationalen Indochina-Konferenz hatten sich Großbritannien, Frankreich, die Philippinen, Thailand, Australien und Neuseeland am 8. September 1954 zur South East Asian Treaty Organization (SEATO) zusammengeschlossen mit dem Hauptziel, die nicht-kommunistischen Staaten in Indochina - Südvietnam, Laos und Kambodscha - vor einer kommunistischen Aggression zu schützen.

478 Der Bagdad-Pakt verband die NATO-Staaten Großbritannien und Türkei seit 1956 mit dem Irak, dem Iran und Pakistan. Aufgrund des Umsturzes in Bagdad am 14. Juli 1958 war der Irak ausgeschieden.

479 Das war der Charakter, den die UdSSR insbesondere der Politik des amerikanischen Außenministers John Foster Dulles zuschrieb. 
hen Beschäftigungsstandes der Bevölkerung in der Industrie sieht, will man das ausschließen; und es wird ausgeschlossen, weil das darauf hinausläuft, und die Leute werden es begreifen, und die Leute begreifen jeden Tag mehr das Fatale dieser Politik und werden die Regierungen zur Beendigung des Wettrüstens zwingen. Gibt es denn jetzt keine Möglichkeit, statt der Produktion von Vernichtungsinstrumenten in großem Umfang die Produktion von Konsumgütern und Produktionsanlagen zu organisieren? Und wenn jetzt die Monopolisten der Vereinigten Staaten von Amerika, ihre Produktion von Konsumgütern an andere Länder verkaufen. ${ }^{480}$ Schließlich ist es den Monopolisten egal, in welche Hände diese Güter kommen, und daher geben sie sie an andere Staaten ab, die ihre Politik, die Politik des Kalten Krieges unterstützen. Sie brauchen sie [die Konsumgüter] umso mehr auf, sie unterminieren die Entwicklung ihrer Industrie[produktion] und machen sie damit von ihrem Monopolkapital abhängig und ziehen daraus Vorteil und halten diese Länder in einem bettelarmen Zustand. Stattdessen würde die Menschheit aufatmen, wenn Konsumgüter hergestellt würden. Und der Markt, dem sie jetzt Waffen liefern, ist dafür unbegrenzt aufnahmefähig. Und wenn sie Anlagen bekämen für die Förderung von Erdöl, für Prospektierungen, für die Verarbeitung ihrer Bodenschätze, für den Aufbau ihrer eigenen verarbeitenden Industrie zur Produktion von Konsumgütern, würde das den Aufschwung der Wirtschaft, des Lebensstandards und den Aufschwung der Kultur der Völker dieser Länder fördern. Wessen bedarf es dazu? Es ist auf diese Politik zu verzichten und der Standpunkt einer Politik der friedlichen Koexistenz und der Entwicklung des Handels einzunehmen. Und diese Politik würde allen Ländern Vorteile bringen, weil beim Blick auf die Länder der Welt Länder wie Indien, Länder wie China, Pakistan, Indonesien, Iran und andere Länder des Arabischen Orients [sowie] die Sowjetunion eine unwahrscheinliche Masse von Produktionsanlagen und Konsumgütern aufnehmen könnten. Daher würde hier keine Vergrößerung der Armee der Arbeitslosen drohen, sondern es würde umgekehrt zur beträchtlichen Vergrößerung der Konsumgüterproduktion beitragen, und das würde einen höheren Lebensstandard schaffen und zum Aufschwung der Produktion, der Wirtschaft und der Kultur beitragen.

- Wo er von der Beschäftigung spricht, sollte gesagt werden, dass dafür natürlich nicht Maßnahmen ergriffen werden sollten, die diese Entwicklung fördern würden. Eine der wichtigsten Maßnahmen, die unter diesen Umständen zu verwirklichen ist, besteht darin, die Preise für Konsumgüter zu senken und die Löhne der Werktätigen zu erhöhen. Das würde die Kaufkraft der Bevölkerung erhöhen, zur Hebung des Lebensstandards beitragen und Voraussetzungen für die Produktion einer größeren Menge von Konsumgütern schaffen, welche die Bevölkerung benötigt.

- Schließlich werden gewisse Mittel für die Rüstung ausgegeben. Wenn man diese Mittel für dieses und jenes ausgibt, wird das die Bedingungen für einen Aufschwung schaffen usw.

- Und es ist angebracht zu bemerken, wer hat irgendeinem Land das Recht gegeben, eine Doktrin für die einen oder anderen Länder aufzustellen außer dem

480 Unvollendeter Satz. 
Recht der Willkür gestützt auf Gewalt? Doch die Zeiten sind jetzt vorbei, als dieses „Recht“ faktisch als Recht galt.

- Vielleicht sollte man hier dazu sagen, dass derartige Dinge gemacht werden unter Verletzung des Völkerrechts und [dass] die UNO, die darauf reagieren müsste, untätig bleibt, die Doktrin aber klar das Ziel verfolgt, was auch direkt zum Ausdruck gebracht wird: Einmischung in die Angelegenheiten anderer Staaten, was vom Statut und von der Ordnung der UNO verurteilt wird.

- Hauptsächliche Bedeutung hat die große Menge an schöpferischen Kräften des eigenen Volkes. Die Hauptsache sind die Menschen in den Ländern.

- Vielleicht sollte ich sowohl in meiner Erklärung als auch hier den Gedanken anführen, dass zur gleichen Zeit, da die Länder des Warschauer Vertrages eine Reduzierung ihrer Streitkräfte und Rüstungsausgaben vornehmen und die uns gegenüberstehenden Länder der NATO dazu aufrufen, diese und insbesondere die Vereinigten Staaten von Amerika, statt das zu erwidern und unserem Beispiel zu folgen, eine Politik der Vergrößerung der Truppen, der Rüstungen und der Rüstungsausgaben betreiben und sie damit eine Politik betreiben, die nicht zur Entspannung und Verständigung zwischen den Staaten beiträgt, um die Koexistenz zu gewährleisten, sondern im Gegenteil wollen sie damit unsere Länder dazu veranlassen, zum Wettrüsten zurückzukehren und es zu forcieren und den Kalten Krieg zu entfachen. Daher müssen die Völker wachsam sein und ihre Aktivität im Kampf gegen die Kräfte erhöhen, die auf eine Vorbereitung des Krieges hinwirken. (Für die Deklaration und für die Erklärung).

Übersetzt aus dem russischen Originaltext RGANI, fond 52, opis' 1 , delo 443, Bl. 136-145

\section{Gespräch Chruschtschows mit dem Politiker der britischen Labour Party K[onni] Zilliacus481 am 4. September 1958}

Zilliacus sagt zu Beginn des Gesprächs, dass er das Gespräch lieber ohne Dolmetscher führen will, in russischer Sprache, weil das einen engeren Kontakt zwischen den Gesprächspartnern herstellt. N. S. Chruschtschow ist damit einverstanden. Zilliacus dankt N. S. Chruschtschow für den Empfang und übermittelt von $\mathrm{A}$ [neurin] Bevan einen „sehr herzlichen Gruß“. Weiter erläutert Zilliacus das Ziel seines Besuches in der Sowjetunion.

Zilliacus: Ich möchte mit Deputierten des Obersten Sowjets und anderen Vertretern des sowjetischen Staates inoffiziell die Frage erörtern, inwieweit sich im Bereich der Außenpolitik die Positionen der Sowjetunion den Positionen der Labour Party annähern. Wir haben den Eindruck gewonnen, dass wir in diesem Bereich viel mit Ihnen gemeinsam haben.

481 Konni Zilliacus war neben Aneurin Bevan der führende Vertreter des linken Flügels in der Labour Party. 